\title{
Struggle for Space: Viral Extinction through Competition for Cells
}

\author{
José A. Cuesta, ${ }^{1}$ Jacobo Aguirre, ${ }^{2}$ José A. Capitán, ${ }^{1}$ and Susanna C. Manrubia ${ }^{2}$ \\ ${ }^{1}$ Grupo Interdisciplinar de Sistemas Complejos (GISC), Departamento de Matemáticas, \\ Universidad Carlos III de Madrid, Leganés, Madrid, Spain \\ ${ }^{2}$ Centro de Astrobiología, CSIC-INTA, Torrejón de Ardoz, Madrid, Spain
}

(Received 8 September 2010; published 14 January 2011)

\begin{abstract}
The design of protocols to suppress the propagation of viral infections is an enduring enterprise, especially hindered by limited knowledge of the mechanisms leading to viral extinction. Here we report on infection extinction due to intraspecific competition to infect susceptible hosts. Beneficial mutations increase the production of viral progeny, while the host cell may develop defenses against infection. For an unlimited number of host cells, a feedback runaway coevolution between host resistance and progeny production occurs. However, physical space limits the advantage that the virus obtains from increasing offspring numbers; thus, infection clearance may result from an increase in host defenses beyond a finite threshold. Our results might be relevant to devise improved control strategies in environments with mobility constraints or different geometrical properties.
\end{abstract}

DOI: 10.1103/PhysRevLett.106.028104

PACS numbers: 87.23. $-\mathrm{n}, 87.10 .-\mathrm{e}, 87.18 .-\mathrm{h}$

One of the common strategies used by viruses to ensure fast adaptation is the steady production of mutants and of enormous amounts of progeny, two characteristics that challenge the design of therapies able to induce viral extinction. Increased mutagenesis has been successfully used in vitro to eradicate infectivity, though there is no general agreement on the pathways through which extinction supervenes [1-4]. In mutational meltdown, the population becomes extinct because the average growth rate falls below one, through a process different from the error threshold predicted by classical quasispecies models [5] or from stochastic extinction of infectivity [6,7]. The progress of an infection is further conditioned by the geometry of the space where it occurs [8-10]. Experiments with viruses infecting bacteria have highlighted differences in the internal structure of populations evolving in liquid medium or on bacterial monolayers [11]. These results might give clues to treat viral infections of two-dimensional tissues: It has been shown that space induces a remarkably strict clustering of the propagation in crops [12], in leaves [13], and in lawns of cells [14]. Advances in therapy design ask for an improved understanding of the mechanisms causing viral extinction before they can be applied in vivo.

Models of viral evolution necessarily make simplifying assumptions, and real virus behavior often deviates substantially from their predictions [15]. Current quasispecies models implement high mutation rates that yield heterogeneous populations - a property of most viruses infecting plants [16] and of many viral populations on cellular monolayers [17]. One common approximation is to assume that all new mutations have a deleterious effect on fitness, thus neglecting beneficial and neutral mutations. However, adaptation occurs frequently, and even at low population numbers. Classical quasispecies models assumed the existence of a unique master sequence of high fitness, while we currently know that a huge amount of different sequences yield phenotypes that perform equally well. When beneficial and neutral mutations are considered, extinction might supervene through mechanisms other than error thresholds [4]. The predictions yielded by models of viral evolution depend on whether physical space is explicitly considered or not [18]. In quasispecies models, cell-to-cell transmission or local transport of viruses unavoidably results in a lower value of the critical error threshold $[19,20]$. Clusterization of viral types is responsible for the latter effect, as well as for enhancing the coexistence of types in the quasispecies $[10,21]$.

In this Letter we introduce a model representing the propagation of infections on two-dimensional arrays of cells. We incorporate two realistic features of infection propagation often disregarded in quasispecies models: a non-negligible fraction of beneficial mutations and a host resistance to infection. We show that with spare susceptible cells at each generation, the virus can escape host resistance by increasing its progeny. However, when viruses compete for infecting cells, increasing the progeny does not pay and infection clearance occurs at a finite value of the host resistance. In this case the model boils down to a multicomponent generalization of the Domany-Kinzel (DK) probabilistic cellular automaton [22] hence classifying viral extinction within the directed percolation (DP) universality class, alongside propagation of immiscible fluids in porous media and other similar problems.

In the model, a viral population is described as an ensemble of (pheno)types, each characterized by a replicative ability $r=0, \ldots, R$ standing for the number of offspring able to infect cells produced by each type under replication. Deleterious or beneficial mutations decrease or increase (with probabilities $p$ and $q$, respectively) offspring's replicative ability in one unit. Susceptible cells have a resistance 
to infection $\pi$; i.e., a viral particle can infect a cell with probability $1-\pi$. For simplicity, we consider that the dynamics proceeds in discrete generations.

Assume first that the availability of susceptible cells is large enough for every viral particle with $r>0$ to actually meet a cell to infect, no matter its replicative ability $r$. This is a mean-field dynamical description, where infection occurs with a probability $1-\pi$. If $n_{r}(t)$ denotes the number of viral particles of type $r$ at generation $t$, for $1 \leq$ $r<R$

$$
\begin{aligned}
n_{r}(t+1)= & (1-\pi)\left[r(1-p-q) n_{r}(t)\right. \\
& \left.+(r+1) p n_{r+1}(t)+(r-1) q n_{r-1}(t)\right], \\
n_{R}(t+1)= & (1-\pi)\left[R(1-p) n_{R}(t)\right. \\
& \left.+(R-1) q n_{R-1}(t)\right] .
\end{aligned}
$$

Additionally, there is a class $r=0$ which has lost its replicative ability and is maintained by class $r=1$ through deleterious mutations, i.e., $n_{0}(t+1)=(1-\pi) p n_{1}(t)$. As the initial condition, we take $n_{r}(0)=\delta_{r, R}$.

An exact analytic solution exists for the case $q=0$, which represents the worst situation for the survival of the virus: if extinction does not occur for $q=0$, it will not occur for $q>0$ either. In the limit $t \rightarrow \infty$, the population of every class grows at a fixed rate, $n_{r}(t+1)=$ $\lambda n_{r}(t)$. System (1) then becomes an eigenvalue problem, $\lambda$ being its largest eigenvalue. For $q=0$, the asymptotic growth rate is $\lambda=(1-\pi) R(1-p)$ and the fraction of virus in class $r$ is

$$
x_{r} \equiv \lim _{t \rightarrow \infty} \frac{n_{r}(t)}{\sum_{r=0}^{R} n_{r}(t)}=\left(\begin{array}{c}
R \\
r
\end{array}\right) p^{R-r}(1-p)^{r} .
$$

Extinction occurs when $\lambda \leq 1$, i.e., if the maximum progeny production $R \leq R_{c}=(1-\pi)^{-1}(1-p)^{-1}$. Although the larger the resistance to infection of the cell $\pi$, the more demanding becomes this condition, $R$ can always be increased beyond $R_{c}$ to avoid extinction. If the number of susceptible cells is unlimited and increasing $R$ always improves the chances of survival of the pathogen, a runaway coevolution between the virus and the host is to be expected.

The case $q=0$ is not representative of the situation when $q>0$ though. The stationary distribution (2) has a well-defined $R \rightarrow \infty$ limit, whereas the permanent improvement allowed when $q>0$ forbids a steady state in the same limit. As a matter of fact, it can be shown that in that case the viral population grows superexponentially [23].

Consider now a restricted number of susceptible cells available. This occurs when cells have a spatial distribution and/or when the mobility of viruses is limited. To assess the consequences of this constraint, we study the dynamics proceeding on a triangular lattice with periodic boundary conditions. Even if infection starts off from a single infected cell, the propagating front becomes asymptotically flat [10]. Hence, we consider that cells form arrays in one spatial dimension, and infection advances one row of cells per generation. Let $r_{i}(t)$ be the replicative ability of the individual occupying site $i=1, \ldots, L$ at generation $t$.

To account for the limited mobility of the viral particles, they are constrained to infect only neighboring cells. Hence, at generation $t+1$ site $i$ will be occupied by one of the offspring of the individuals at sites $i$ or $i+1$ at the parental generation $t$. The probability that $r_{i}(t+1)=r$, given that $r_{i}(t)=r_{1}$ and $r_{i+1}(t)=r_{2}$, is

$$
P\left(r \mid r_{1}, r_{2}\right)=\pi^{r_{1}+r_{2}} \delta_{r, 0}+\left(1-\pi^{r_{1}+r_{2}}\right) \frac{r_{1} p_{r_{1}, r}+r_{2} p_{r_{2}, r}}{r_{1}+r_{2}},
$$

where $p_{r, r-1}=p$ for $r \leq R, p_{r, r+1}=q$ and $p_{r, r}=1-$ $p-q$ for $r<R, p_{R, R+1}=0$, and $p_{R, R}=1-p$. We take $r_{i}(0)=R$ for all $i=1, \ldots, L$ when the infection begins. According to (3), the probability that the cell resists the viral attack is given by $\pi^{r_{1}+r_{2}}$, i.e., the probability that infection fails after $r_{1}+r_{2}$ independent trials. With the complementary probability $1-\pi^{r_{1}+r_{2}}$ infection occurs, and one individual randomly drawn among the $r_{1}+r_{2}$ possible occupies the site. Its replicative ability may change due to mutations as specified. The limit case $\pi \rightarrow 1$ describes an immune host, whereas in the limit $\pi \rightarrow 0$ the host plays no role in the progress of infection. Two relevant quantities that characterize the dynamical state of the system are the spatial density $\rho(t)$ of virus with $r \geq 1$ at time $t$ and the average replicative ability of the population $\bar{r}(t)=$ $(\rho(t) L)^{-1} \sum_{i=1}^{L} r_{i}(t)$. A systematic study of these two quantities in the limit $t \rightarrow \infty$ as a function of $p$ and $\pi$ portrays the asymptotic behavior of the model.

In the absence of beneficial mutations $(q=0)$, the class with the highest $r$ present disappears when $p$ or $\pi$ increase beyond a threshold value. These transitions are somehow analogous to the error threshold described for simple models of quasispecies [5], where the master sequence disappears and the population turns into a cloud of low-fitness replicating mutants. For constant resistance to infection $\pi$, there is a hierarchy of domains in the phase space where high- $r$ classes are sequentially lost as $p$ increases (see Fig. 1). For increasing $\pi$, the largest $r$ value present at global extinction increases.

There are two features of Fig. 1 for $q=0$ which are amenable to further analysis. The first one is the last error threshold, when class $r=1$ disappears. There is a mapping of this transition to the DK cellular automaton. When only classes $r=1$ and $r=0$ are present, sites are either replicative (occupied) or nonreplicative (empty), yielding probabilities (3) $P(1 \mid 0,0)=0, p_{1} \equiv P(1 \mid 1,0)=P(1 \mid 0,1)=$ $(1-\pi)(1-p)$, and $p_{2} \equiv P(1 \mid 1,1)=\left(1-\pi^{2}\right)(1-p)$, which define a DK automaton. Curve $C$ in Fig. 1 coincides with the DK transition cast in the variables $(p, \pi)$ describing our model. This curve meets the $\pi=0$ axis at $p_{c}=$ $0.294510(6)$, a value derived from the known site percolation critical point [24].

The second feature obtained from a mapping with the DK model is the extinction threshold of the highest class $r=R$ 


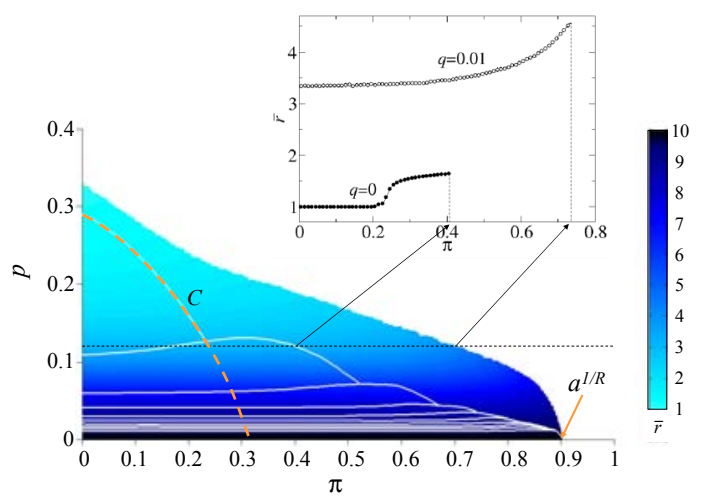

FIG. 1 (color online). Phase space of the model with $R=10$ and two choices for $q: q=0$, where the highest- $r$ classes are sequentially lost as $p$ increases through transitions analogous to the error threshold of Eigen's quasispecies model (white lines), and $q=0.01$, where only global extinction of the population is possible. Color code for the average replicative ability $\bar{r}$ when $q=0.01$. In the white domain, infection is cleared in finite time. The top extinction occurring for $q=0$ can be obtained from that of the DK cellular automaton (curve $C$ ). The inset shows $\bar{r}$ for $p=0.11$ and two values of $q$. Data have been obtained with a system of size $L=10^{4}$ and averaging over $5 \times 10^{3}$ time steps after a transient of $2 \times 10^{4}$ generations.

at $p=0$. Since the initial state only contains individuals of this type, no other types will ever appear because of the lack of deleterious mutations. Hence, sites can be either occupied or empty, just as in the DK model. Transition probabilities correspond to the bond percolation case, from which it follows that the threshold occurs at $\pi_{c}=a^{1 / R}$, with $a=0.3552998(9)$. Only in the limit $R \rightarrow \infty$ would $\pi_{c} \rightarrow 1$, taking a lower value for any other combination of parameters.

For $p \neq 0$ and $q>0$, no matter how small, all replicative types are simultaneously present in the infective phase (see Fig. 1). For a fixed value of $\pi$, lower $r$ classes get more populated upon increasing $p$; hence, the average replicative ability decreases. The behavior as $\pi$ increases at constant $p$ is of a different nature. As $\pi$ increases, infected cells become sparser. In the absence of neighbors, the probability that a cell at generation $t+1$ is infected by offspring of an individual of class $r$ at generation $t$ is proportional to $\left(1-\pi^{r}\right)$ This quantity more severely penalizes low- $r$ classes as $\pi$ increases, such that high- $r$ classes receive a relative advantage and $\bar{r}$ increases as $\pi_{c}$ is approached. At the critical value $\pi_{c}$ the quasispecies eventually collapses because propagation is prevented by host defenses (see the inset of Fig. 1).

There is another relevant difference between the two models. As we have seen, when cells are in excess any host resistance $\pi$ can be circumvented by increasing $R$. In contrast, when viral classes have to compete for susceptible cells, there is a critical value of $\pi$ above which this is no longer true. The reason (illustrated by Fig. 2) is that the average replicative ability $\bar{r}$ quickly saturates to a constant value upon increasing $R$. In other words, when viruses

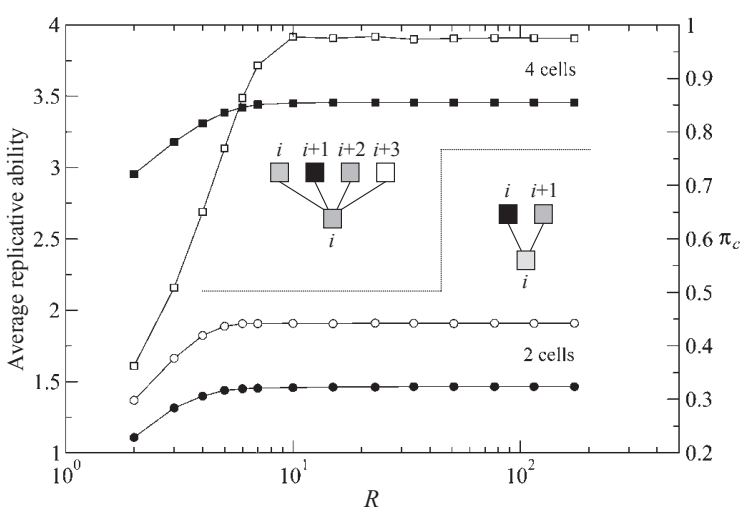

FIG. 2. When the number of susceptible cells available is limited, the average replicative ability $\bar{r}$ (open symbols, left $y$ axis) and the critical value of the resistance to infection $\pi_{c}$ (solid symbols, right $y$ axis) saturate to finite values as $R$ increases. In these curves, $p=0.2, q=0.01$, and averages over 50 independent realizations for each value of $\pi_{c}$ have been performed. Meansquare deviations are smaller than symbol size.

struggle for infecting a limited number of cells, increasing $R$ does not provide any additional advantage. In order to show that this effect results from the limitation in the number of cells available, we have modified the model to consider a case where four cells are available for each infection event. This amounts to increasing the competition between types, such that low- $r$ classes are at a disadvantage with respect to the previous situation and $\bar{r}$ increases [10] (see Fig. 2). As a side effect, the critical value of the cell resistance, $\pi_{c}$, at which extinction occurs also saturates to a value below 1 as $R \rightarrow \infty$. A runaway coevolution is therefore excluded in this case: if the host cell is able to increase its defenses beyond $\pi_{c}$, the infection will not progress.

When the number of susceptible cells is limited, the transitions to extinction belong to the universality class of DP for any $q \geq 0$. For $q=0$ we have rigorously proven this for the extinction of the $r=1$ class as well as when $p \rightarrow 0$, by mapping the model to a DK cellular automaton, known to belong to the DP class [24]. In addition, our model is defined on a set of local rules, has no special symmetries or disorder, and exhibits a continuous transition to a unique absorbing state (total extinction). Therefore, according to the DP conjecture it should belong to the DP universality class. An empirical proof is provided in Fig. 3, where $\rho(t)$ is appropriately rescaled according to the exponents of DP. The transition point $p_{c}$ is numerically determined as the value of $p$ yielding the best collapse. Finally, the study of the variation of $\rho(t)$ at $p_{c}$ with the system size $L$ is characterized by the dynamic exponent $z=1.580745(10)$. This analysis has been repeated for the transition where $r=2$ disappears at $q=0$, with analogous results.

Formally, the probabilistic model presented here defines a multicomponent generalization of the DK cellular automaton. Our model only focuses in a limited region of its parameters in which an active-inactive transition $(q>0)$ or several $(q=0)$ occur with the criticality of directed percolation. Exploring in depth the whole phase 


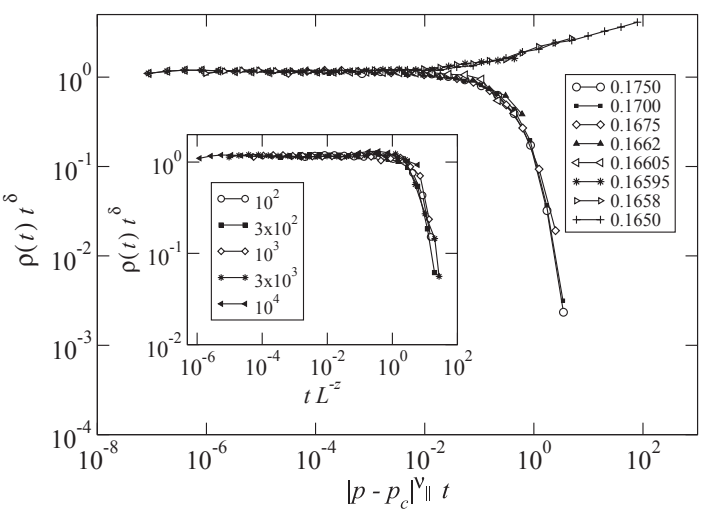

FIG. 3. Data collapse of curves $\rho(t)$ according to the critical exponents known to characterize $(1+1)$ directed percolation, which are $\delta=0.159464(6), \quad \nu_{\|}=1.733847(6)$, and $z=$ $1.580745(10)$ [24]. Parameters in these simulations are $R=2$, $q=0.01, \pi=0.3$. The system size is $L=10^{4}$ for the main plot, where the legend shows the values of $p$ for each curve. For this choice the transition occurs at $p_{c}=0.16600$ (3). Legend in the inset gives the values of the system size $L$. Data have been averaged over 50 independent realizations and up to $10^{8}$ generations for each curve.

space of this model in search for deviations of this behavior is an interesting open question left out by the present study.

Our model could be modified to better mimic other biological situations. In many plants, different individuals display variable degrees of resistance to viral infection [25], a case that could be implemented by introducing a host-dependent $\pi$. This would introduce a form of quenched spatial disorder that may lead to universality classes other than DP [24]. Analogous changes would be effected by temporally quenched disorder, a situation holding, e.g., when susceptibility to infection depends on the age of the host. An example of the latter is systemic acquired resistance, a durable form of immunization observed in plants [26]. Spatially quenched disorder could change the properties of viral extinction to those of dynamic percolation [27]. Current knowledge on the properties of percolation phenomena in a variety of different situations might inspire unprecedented strategies to stop infection propagation in those different environments.

The hindrance to the viability of a virus brought about by a limited availability of cells has some empirical support. While all plant viruses infect the tissue that carries nutrients through the vascular system (phloem), where mobility is high and susceptible cells are abundant, some viruses (e.g., luteoviruses and some geminiviruses [28]) are not able to infect leaves, where mobility is limited to cell-to-cell transmission and fewer uninfected cells are available per infective cycle. The reverse situation is as yet unknown.

The authors acknowledge conversations with E. Lázaro and F. García-Arenal and the support of the Spanish
MICINN under projects FIS2008-05273 and MOSAICO and of Comunidad de Madrid (Spain) under project MODELICO-CM.

[1] C. O. Wilke, BMC Evol. Biol. 5, 44 (2005).

[2] J. J. Bull, L. A. Meyers, and M. Lachmann, PLoS Comput. Biol. 1, e61 (2005).

[3] N. Takeuchi and P. Hogeweg, BMC Evol. Biol. 7, 15 (2007).

[4] S. C. Manrubia, E. Domingo, and E. Lázaro, Phil. Trans. R. Soc. A 365, 1943 (2010).

[5] M. Eigen, Naturwissenschaften 58, 465 (1971).

[6] A. Grande-Pérez, E. Lázaro, P. Lowenstein, E. Domingo, and S.C. Manrubia, Proc. Natl. Acad. Sci. U.S.A. 102, 4448 (2005).

[7] J. Iranzo and S. C. Manrubia, Europhys. Lett. 85, 18001 (2009).

[8] T. Petermann and P.D.L. Ríos, J. Theor. Biol. 229, 1 (2004).

[9] Dynamical Processes on Complex Networks, edited by A. Barrat, M. Barthélemy, and A. Vespignani (Cambridge University Press, Cambridge, England, 2008).

[10] J. Aguirre and S.C. Manrubia, Phys. Rev. Lett. 100, 38106 (2008).

[11] C. Cases-González, M. Arribas, E. Domingo, and E. Lázaro, J. Mol. Biol. 384, 1120 (2008).

[12] B. A. Coutts, M.L. Thomas-Carroll, and R. A. C. Jones, Ann. Appl. Biol. 145, 339 (2004).

[13] T. Takahashi, T. Sugawara, T. Yamatsuta, M. Isogai, T. Natsuaki, and N. Yoshikawa, Phytopathology 97, 1200 (2007).

[14] Y. Lee and J. Yin, Nat. Biotechnol. 14, 491 (1996).

[15] M. Eigen, Proc. Natl. Acad. Sci. U.S.A. 99, 13374 (2002).

[16] F. García-Arenal, A. Fraile, and J. M. Malpica, Annu. Rev. Phytopathol. 39, 157 (2001).

[17] S. C. Manrubia and E. Lázaro, Phys. Life Rev. 3, 65 (2006).

[18] D. A. Rand, M. Keeling, and H. B. Wilson, Proc. R. Soc. B 259, 55 (1995).

[19] S. Altmeyer and J. S. McCaskill, Phys. Rev. Lett. 86, 5819 (2001).

[20] S. Toyabe and M. Sano, Physica (Amsterdam) 203D, 1 (2005).

[21] R. Pastor-Satorras and R. V. Solé, Phys. Rev. E 64, 51909 (2001).

[22] E. Domany and W. Kinzel, Phys. Rev. Lett. 53, 311 (1984).

[23] J. A. Cuesta, Math. Comput. Modell. (in press).

[24] H. Hinrichsen, Adv. Phys. 49, 815 (2000).

[25] B.-C. Kang, I. Yeam, and M. M. Jahn, Annu. Rev. Phytopathol 43, 581 (2005).

[26] W.E. Durrant and X. Dong, Annu. Rev. Phytopathol 42, 185 (2004).

[27] P. Grassberger, Math. Biosci. 63, 157 (1983).

[28] Fields Virology, edited by D. M. Knipe and P. M. Howley (Lippincott, William \& Wilkins, Philadelphia, PA, 2007), Vol. I, 5th ed. 